\title{
Caractérisation et renforcement des sols avec inclusion de nappes plastiques souples discontinues
}

T. MESSAS

L2M, IUT A Génie Civil,

Lyon 1

43, bd du 11-Novembre 1918

69622 Villeurbanne Cedex
La production des déchets de matières plastiques ne cesse de croítre. On peut valoriser ces déchets en les réemployant pour renforcer les sols et améliorer leurs caractéristiques mécaniques. Pour obtenir la meilleure représentativité des phénomènes réels, les essais de laboratoire (frottement, ancrage, cisaillement) ont été effectués sur des appareils de grandes dimensions. Ils ont permis de mesurer l'influence de deux paramètres : la taille et la masse surfacique des éléments discontinus de renfort.

Soils characterization and reinforcement with discontinous plastic materials

The production of plastic materials waste increases continuously. It is possible to valorize these wastes by its reuse as soils reinforcement. On the other hand, this technique increases the mechanical characteristics of soils. A best representativity of real phenomena was obtained by laboratory tests (friction, anchorage, shear) carried out on big size apparatus. Tests carried out by this new shear apparatus permited us to observe the influence of two parameters : the size and the surfacic load of this reinforcement discontinuous elements. 


\section{Introduction}

L'amélioration des caractéristiques mécaniques du sol, support de tout ouvrage de génie civil, est une préoccupation des géotechniciens. Une technique de renforcement des sols consiste à adjoindre à ces derniers des inclusions dans le but d'améliorer leurs propriétés mécaniques:

Le renforcement des sols par les inclusions souples (non tissées) a été proposé par Leflaive et Pring (1974) et McGolon (1977). Une optimisation du renforcement est possible pour faire évoluer les règlements et diminuer les couts, tout en garciant le même niveau de sécurité.

Les déchets de matières plastiques (sacs d'emballage, bouteilles...), qui connaissent une augmentation sensible liée à leur utilisation diversifiée, peuvent être utilisés comme matériaux de renforcement. Ces déchets posent de nombreux problèmes car leur recyclage nécessite des tris minutieux, leur élimination par incinération est un danger pour l'environnement, et leur mise en décharge est coûteuse.

Pour aider à résoudre ces problèmes, Coulet et al, (1987) ont proposé un procédé de renforcement géotechnique basé sur l'association de déchets de plastique et de terre qui permet d'améliorer les caractéristiques mécaniques des sols, et constitue une solution économique et écologique aux problèmes posés par les dêchets.

Depuis sa mise en œuvre, ce procédé n'a fait l'objet que de quelques essais in situ de stabilité d'un remblai. Malgré les nombreuses expériences au triaxial (Dali et al., 1989), ce domaine n'est jusqu'à présent pas totalement maîtrisé. En effet, les essais déjà effectués n'ont pas permis de rendre compte de l'apport effectif du renforcement. C'est dans cette perspective que s'inscrit le présent travail qui est consacré à la mise en cuvre d'essais de laboratoire (ancrage, frottement, cisaillement). On note que la plupart des techniques de renforcement utilisent des matériaux qui possèdent une continuité soit de surface (géatextile), soit de longueur (terre armèe). Ceux qui sont utilisés ici sont discontinus et formés de morceaux souples enchevêtrés les uns aux autres. Afin de représenter le mode réel de rupture lors d'un glissement d'un remblai, un essai de cisaillement original « à plan de cisaillement vertical n a été mis au point. Un appareil a été conçu et construit. Il permet, d'une part, de faciliter la mise en place des morceaux de plastiques avec des surfaces de recouvrement et, d'autre part, de reproduire assez ficlèlement l'état de contrainte existant dans le sol ainsi que le mode de rupture:

Ce type d'essais présente également des avantages par rapport aux expériences sur ouvrages réels :

- la rapidité et le faible coût :

- le contrôle et l'étude de l'influence des divers paramètres mis en jeu :

- la possibilité de multiplier le nombre d'essais;

- la reproductibilité des essais ;

- l'étude du comportement des ouvrages à la rupture.

En outre, les résultats obtenus à partir de ces essais et de leurs interprétations peuvent servir de base de données en vue d'une modélisation numérique fiable et prédictive.

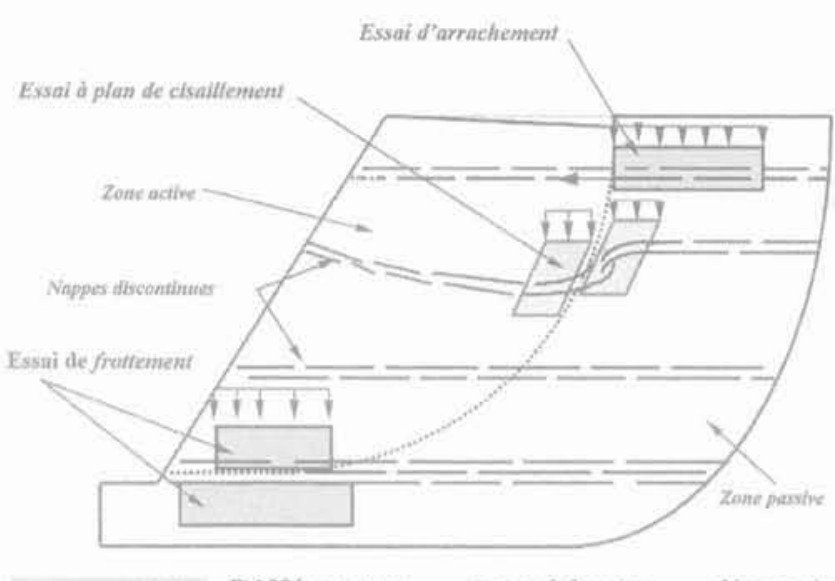

FiG. 1 Différentes procédures d'essais d'interaction mécanique sol-nappes discontinues en matières plastiques.

Different test processus of mechanical soildiscontinuous layers interaction in plastic materials.

\section{2}

\section{Présentation des matériaux et du matériel}

\section{1}

\section{Matériaux}

Le sol utilisé dans le programme d'essais est une grave roulée provenant des carrières de la région lyonnaise, de granulométrie serrée comprise entre $5 \mathrm{~mm}$ et $12 \mathrm{~mm}$. Pour obtenir une bonne reproductibilité des essais, le sol est étuvé pour avoir une teneur en eau nulle. En effet, la teneur en eau ayant une influence sensible sur la cohésion ou sur la densité sèche, il est plus simple de considérer des échantillons secs.

\section{2}

\section{Matières plastiques}

Le renforcement de la grave a été effectué par des nappes de deux types de déchets:

- des feuilles de polyéthylène translucide, de masse surfacique égale à $125 \mathrm{~g} / \mathrm{m}^{2}$ et de résistance de traction à la rupture $1,8 \mathrm{kN} / \mathrm{m}$;

- des déchets de matières plastiques souples constitués essentiellement de sachets d'emballage.

\section{3}

\section{Appareillage d'essai de frottement}

Un appareil permettant d'effectuer des essais bien adaptés à cette étude a été conçu (Fig. 2). Il est composé de deux parties de largeur et de hauteur identiques ( $250 \mathrm{~mm}$ et $200 \mathrm{~mm}$ respectivement). La base d'une longueur de $800 \mathrm{~mm}$ est fixée au sol. La partie supérieure d'une longueur de $600 \mathrm{~mm}$ est longitudinalement mobile, ce qui permet de conserver une surface de cisaillement (ou de frottement) constante au cours de l'essai. La demi-boîte supérieure est déplacée à une vitesse constante égale à $1 \mathrm{~mm} / \mathrm{min}$, et ceci pour tous 
les essais. La traction est exercée par un vérin mécanique d'une force de $50 \mathrm{kN}$. A l'avant, est placé le comparateur (course $100 \mathrm{~mm}$ ) qui permet de suivre le déplacement longitudinal. Un dynamomètre intercalé entre le vérin et la boite permet d'effectuer les mesures des efforts de cisaillement. Le chargement vertical est réalisé au moyen de gueuses de poids différents (1030N, $2100 \mathrm{~N}$...) posées sur un plateau muni d'un piston de section rectangulaire afin de transmettre la charge verticale.

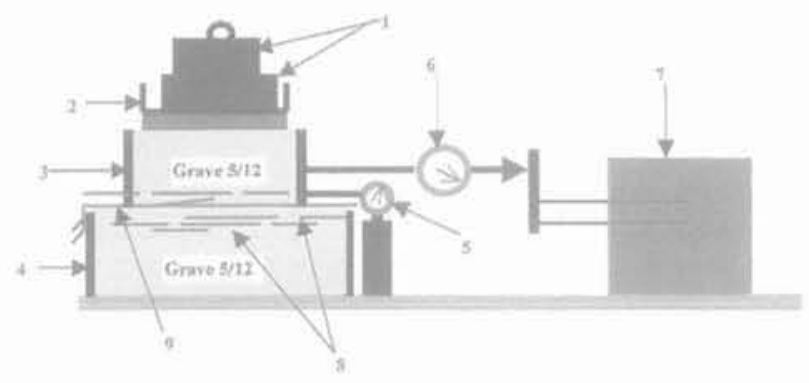

I: Guence da chargenumi

2): Prasaue \&e chargemen:

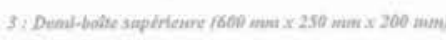

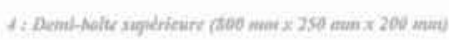

3: Canuparateur

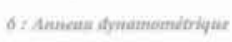

7i Virfin makeariquac

as Nappes discomtinued

2. Nappe coundinue

FIG. 2 Schéma descriptif de Yappareil d'essai de frottement.

Diagram of the experimental device of the friction test.

\section{Appareillage d'essai d'arrachement}

Le but des essais est d'étudier le frottement graveplastique. Pour cela, un modèle de $850 \mathrm{~mm}$ de longueur, $250 \mathrm{~mm}$ de largeur et $200 \mathrm{~mm}$ de hauteur a été construit (Fig. 3). A l'intérieur de celui-ci, la grave est mise en place par couche de $6 \mathrm{~cm}$. Son poids volumique est analogue à celui utilisé lors des essais à la boite de cisaillement $\left(\gamma_{4}=17 \mathrm{kN} / \mathrm{m}^{3}\right)$. Afin de reproduire les mêmes conditions d'essai, on a respecté le même compactage manuel.

Le chargement vertical est réalisé au moyen de gueuses de poids différents (1 $030 \mathrm{~N}, 2100 \mathrm{~N}$...) posées sur un plateau muni d'un piston de section rectangulaire afin de transmettre la charge verticale. Pour extraire le plastique, deux fentes sont réalisées sur les parois frontales. Le système d'arrachement a été mis au point par des essais préliminaires. Au début, le plastique était adjacent au parement (Fig. 4). On a remarqué alors que les grains entraînés par la feuille en traction se coinçaient entre les bords des fentes et la perçait en provoquant la rupture de la feuille. On a donc éloigné la nappe plastique du parement par une plaque de garde (Fig. 5), Les conditions expérimentales sont ainsi voisines de la réalité: La surface du plastique en contact avec le gravier est constante durant tout l'essai d'ancrage. Les nappes plastiques extraites sont pincées entre deux tôles entrant dans la cuve sur une longueur de $0,15 \mathrm{~m}$ et des-

tinées, d'une part, à faciliter la mise en tension de la nappe et, d'autre part, à permettre la suppression des effets de paroi en tête de nappe. Le déplacement de la nappe plastique est effectué à l'aide d'un vérin mécanique de $50 \mathrm{kN}$ et de $50 \mathrm{~cm}$ de course. La vitesse de déplacement pour tous les essais est de $1 \mathrm{~mm} / \mathrm{min}$, c'està-dire en condition quasi statique. La force de traction est mesurée à l'aide d'un dynamomètre. L'essai est poursuivi jusqu'au pic après lequel l'effort de traction décroit d'une façon nette. Le déplacement est mesuré par un grand comparateur (course $100 \mathrm{~mm}$ ).
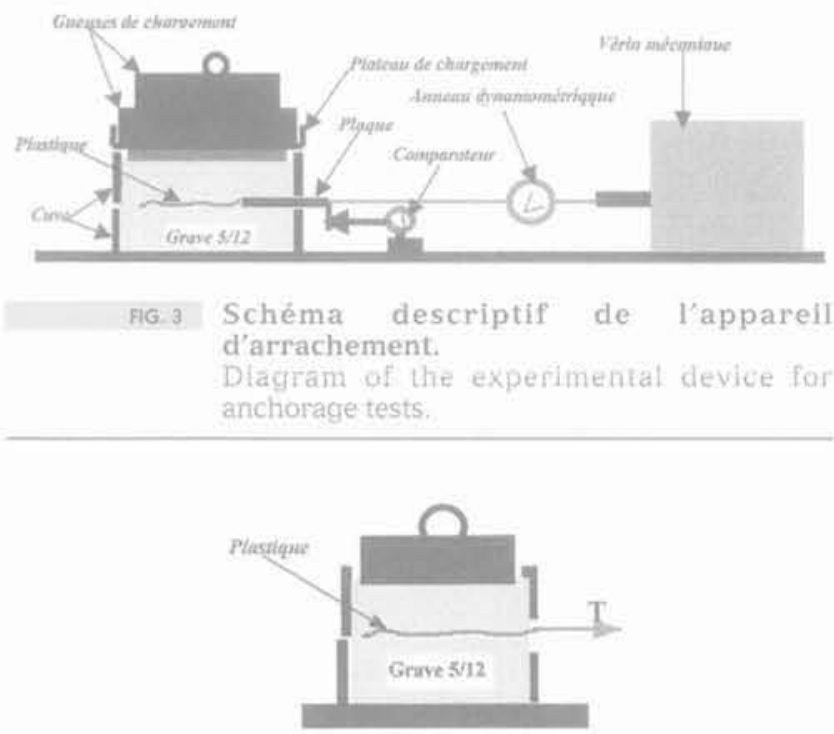

AG. 4 Plastique en parement. Outward plastic.

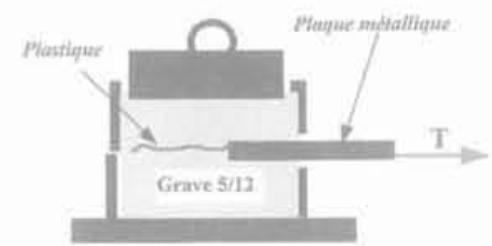

HG 5 Plastique interne. Internal plastic

\section{Appareillage d'essai de cisaillement à plan vertical}

L'appareillage (Fig. 6) est constitué d'une cuve, d'un système de cisaillement et d'un système de chargement vertical du matériau.

Les dimensions de la cuve sont : $600 \mathrm{~mm}$ de longueur, $400 \mathrm{~mm}$ de largeur, $465 \mathrm{~mm}$ de profondeur. Ses parois sont très rigides afin de supprimer toutes déformations. Les parois latérales sont munies de deux fentes pour permettre le passage de l'inclusion. Afin de visualiser les déplacements intérieurs, la paroi frontale est constituée d'une vitre qui résiste à la poussée du sol. La cuve est formée par deux boites de dimensions identiques (300 mm $\times 400 \mathrm{~mm} \times 465 \mathrm{~mm}$ ) dont l'une est fixe et l'autre mobile. Elles sont supportées par des portiques de grandes rigidités permettant d'installer le vérin qui provoque le cisaillement. 
Pour l'ensemble des essais, la vitesse de déplacement est de $1 \mathrm{~mm} / \mathrm{min}$. Un anneau dynamométrique mesure l'effort de cisaillement et un comparateur (course de $100 \mathrm{~mm}$ ) permet de suivre le déplacement vertical. La contrainte normale au plan des feuilles plastiques est donnée par des gueuses accrochées à deux leviers qui appuient sur le sol des deux demi-boites par l'intermédiaire de deux anneaux dynamométriques (Fig, 6).

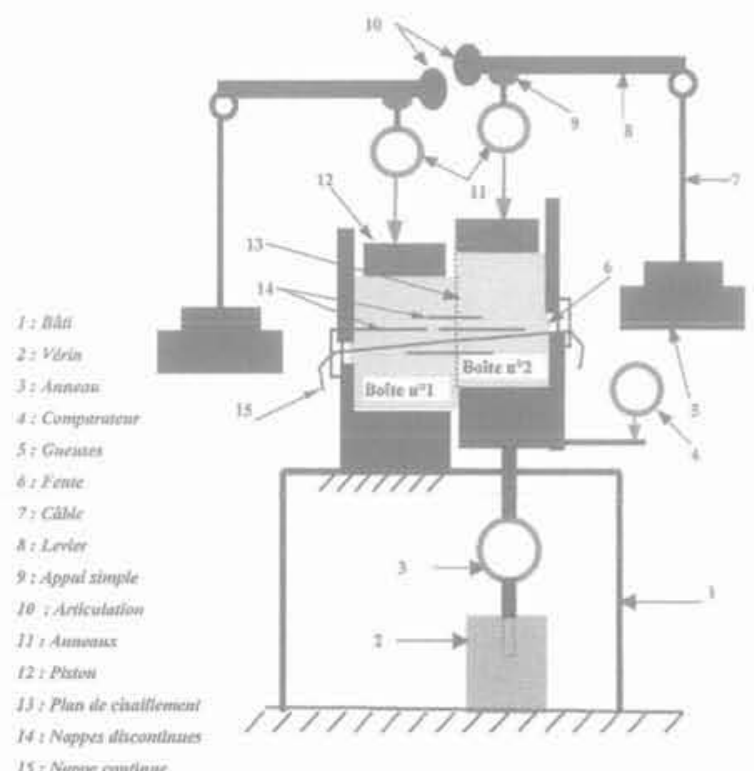

FIG.6 Schẻma de principe du dispositif expérimental de l'essai à plan de cisaillement vertical.

Diacram of the experimental device of the vertical plan shearing test.

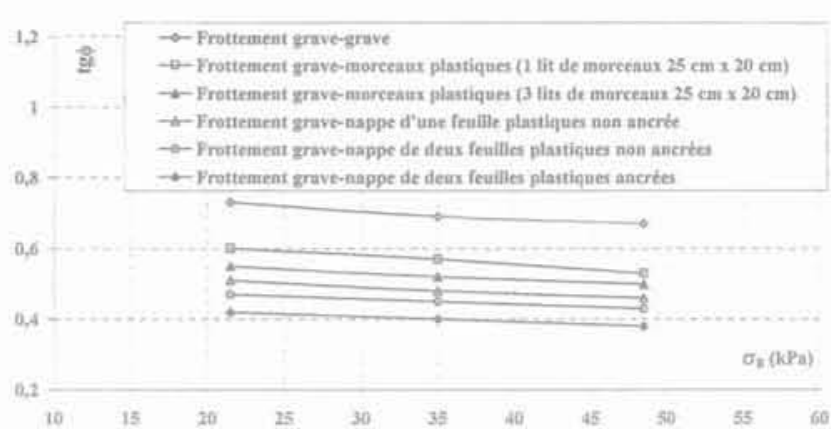

Fic. 7 Évolution du frottement apparent (au pic) avec la contrainte verticale.

Apparent friction evolution versus normal stress.

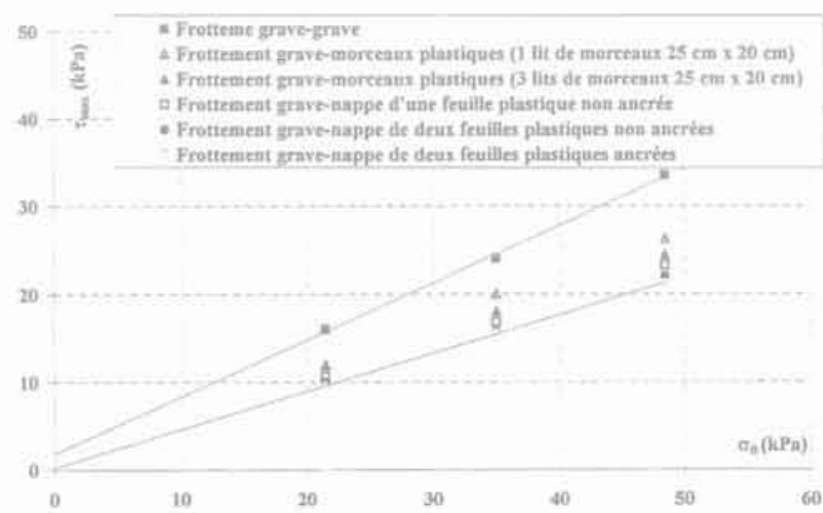

Fic.8 Essai de frottement grave-plastique : contraintes tangentielles maximales. Test of friction gravel-plastic.

\section{Analyse et interprétation des résultats des essais d'ancrage}

\section{Analyse et interprétation des résultats des essais de frottement}

La figure 7, montre que le coefficient de frottement apparent d'interface grave-plastique est plus petit que le frottement apparent de la grave sur elle-même; ce qui est en accord avec de nombreuses études d'interfaces sol-inclusion effectuées par divers chercheurs, Le frottement dépend du sol lui-même (nature, forme de grains) et de l'état de l'interface (lisse ou rugueuse), Le coefficient de l'interface grave-plastique augmente avec la souplesse de cette inclusion. Ceci peut s'expliquer par la non-planéité de la surface de contact graveplastique « effet de festonnage » (J. Bacot, 1981). En conséquence, la surface de rupture n'est pas plane et ne coincide pas avec la surface de séparation entre le sol et le plastique.

L'essai avec nappe non ancrée montre une résistance au frottement $\tau$ inférieure à celle de l'essai avec nappe ancrée (Fig. 8) car la nappe ancrée suit le mouvement des grains du gravier. Les résultats obtenus montrent qu'il y a un écart entre les angles de frottement interne grave-grave et grave-plastiques.

\section{1}

\section{Détermination des paramètres de l'interface sol-plastique}

Afin d'éliminer l'effet de la plaque d'ancrage, des essais complémentaires ont été réalisés sur celle-ci. La contrainte tangentielle grave-plastique est exprimée

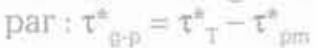

oùr:

$\tau^{*}$, est la contrainte tangentielle totale intégrée à la fois à la résistance apportée par le plastique et à la résistance apportée par la plaque ;

$\tau^{*}$ est la contrainte tangentielle grave-plaque métallique :

$\tau^{*}$ est la contrainte tangentielle grave-plastique.

L'exploitation est effectuée avec les hypothèses suivantes :

- l'effort de traction de la nappe (T) se répartit également entre les deux surfaces (dessus et dessous) de frottement :

- la répartition des contraintes est supposée uniforme sur les surfaces de frottement. 
Comme $S_{\text {dessus }}=S_{\text {dessous., }}$ on peut écrire :

$\tau_{\mathrm{T}}^{*}=\mathrm{T}_{\mathrm{T}} / 2 \mathrm{~S}_{\mathrm{rI}}$

$\tau_{p m}^{*}=T_{p m} / 2 S_{\mathrm{pmm}}$

où :

$T_{T}$ est l'effort d'ancrage total grave-plastique et graveplaque,

$T_{p m}$ est l'effort d'extraction grave-plaque,

$2 \mathrm{~S}_{\mathrm{rT}}$ sont deux surfaces (dessus et dessous) pour le frottement grave-plastiques et grave-plaque métallique, $2 S_{\text {fpm }}$ sont deux surfaces (dessus et dessous) pour le frottement grave-plaque métallique.

Lorsque l'on met deux matériaux en contact, toute tentative de les faire glisser l'un par rapport à l'autre se heurte à l'apparition d'une force de frottement.

On montre qu'il y a proportionnalité entre la contrainte appliquée et la contrainte à fournir pour amorcer le glissement.

$\tau_{\text {(g.p)max }}^{*}=\mu^{*} \sigma=\sigma \operatorname{tg} \phi_{\text {gi- }}^{*}$

où $\mu^{*}=\operatorname{tg} \phi^{*}$ gep est le coefficient de frottement apparent.

\section{2}

\section{Influence de la contrainte normale et de la longueur d'ancrage}

La figure 9 montre que le coefficient de frottement apparent ( $\left.\operatorname{tg} \phi^{*}{ }_{0-p}\right)$ diminue quand la contrainte normale $\left(\sigma_{0}\right)$ augmente. Cette tendance a été mise en évidence par d'autres chercheurs sur les géotextiles.

Pour une contrainte normale donnée, le coefficient de frottement apparent croît avec l'augmentation du nombre des éléments qui forment ces nappes. Ceci peut s'expliquer par le fait que lorsqu'on rassemble les morceaux plastiques, on associe leurs rigidités.

On note que la résistance maximale à l'extraction est proportionnelle à la longueur d'ancrage des morceaux plastiques.

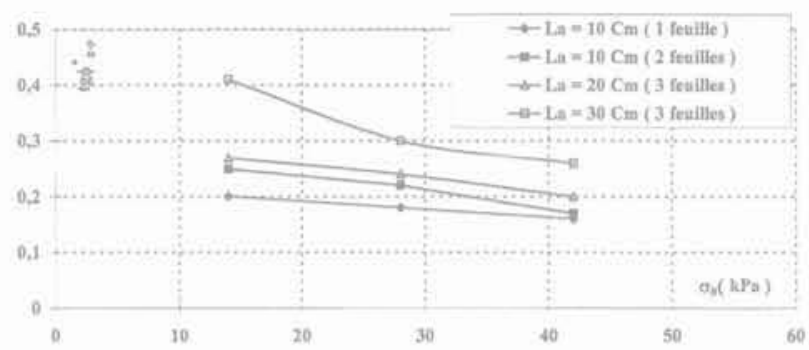

FG.9 Variation du coefficient de frottement apparent en fonction de la contrainte normale.

Variation of the apparent friction coefficient according to the normal stress.

\section{5}

\section{Comparaison entre les essais de frottement et les essais d'arrachement}

Quand on compare la contrainte tangentielle maximale $\left(\tau_{\text {jg pinax }}\right)$ des essais de frottement avec celle des essais d'ancrage ( $\tau^{*}$ (a-pimax $)$, on constate une valeur beau- coup plus importante de $\tau_{\text {(g.pmax }}$ par rapport à $\tau^{*}$ ig-pimax: Ceci peut s'expliquer par la cinématique de l'essai d'ancrage. En effet, les forces de frottement dans cet essai sont mobilisées de façon différente. Le fait de tirer sur la nappe de plastique rend celle-ci plane, ce qui réduit l'effet de son ondulation initiale sur l'augmentation du frottement, chose existante dans l'essai de frottement (effet de festonnage, Bacot, 1981).

On note une certaine analogie qualitative de la variation du coefficient de frottement apparent en fonction de la contrainte normale pour les deux types d'essai.

L'angle de frottement $\phi^{*}$ qui est obtenu lors des essais d'arrachement est inférieur à celui obtenu lors des essais de frottement $\left(\phi^{*}{ }_{g-p}\right.$ est d'environ $23^{\circ}, \phi_{g-p}$ est d'environ $26^{\circ}$ ). On remarque que $\phi^{*}{ }_{q-p}$ correspond à environ deux tiers de l'angle de frottement grave-grave $\left(\phi_{\text {oc }}\right.$ est d'environ $\left.33^{\circ}\right)$. Cette règle est généralement admise dans le dimensionnement des ouvrages renforcés.

Par ailleurs, chacun des essais possède ses particularités et ses avantages, soit pour la facilité de son mode opératoire, soit pour sa représentativité des conditions réelles (in situ). En effet, l'essai de frottement à l'aide de l'appareil de cisaillement a été adapté pour étudier le frottement lié à des propriétés de contact de surface. L'essai d'extraction semble cependant mieux modéliser le comportement réel d'une nappe plastique ancrée.

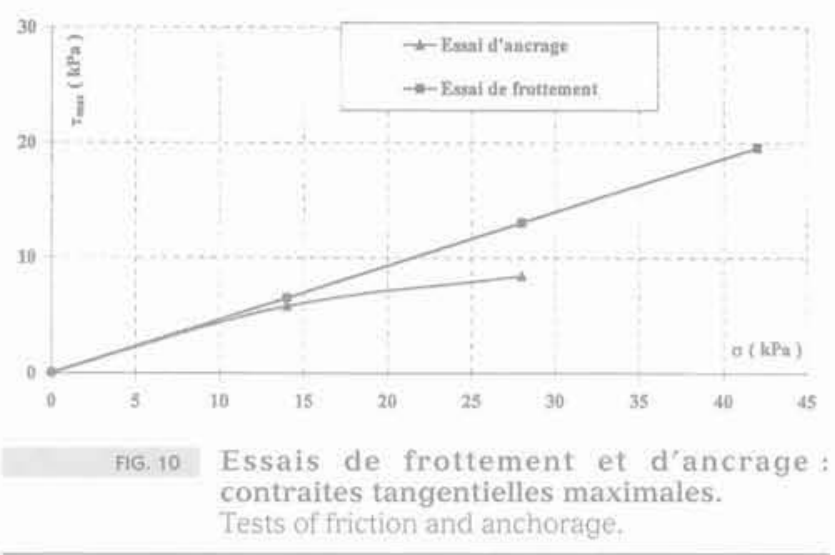
6

\section{Étude de cisaillement du sol renforcé par nappes en matières plastiques}

\section{1}

\section{Renforcement par des nappes continues}

Afin de stimuler un renfort continu dont l'ancrage est parfaitement assuré, les nappes sont fixées à leurs extrémités. La valeur maximale de la contrainte de cisaillement est évidemment obtenue à la rupture des nappes. L'augmentation de la résistance au cisaillement par les nappes de plastique se traduit par l'apparition d'une pseudo-cohésion (Fig. 11), tandis que l'angle de frottement reste constant.

Conformément aux observations faites à partir d'essais triaxiaux ou d'essais de cisaillement à plan horizontal, le frottement apparent (tg $\phi)$ diminue en fonction de la contrainte normale (Fig. 12). 

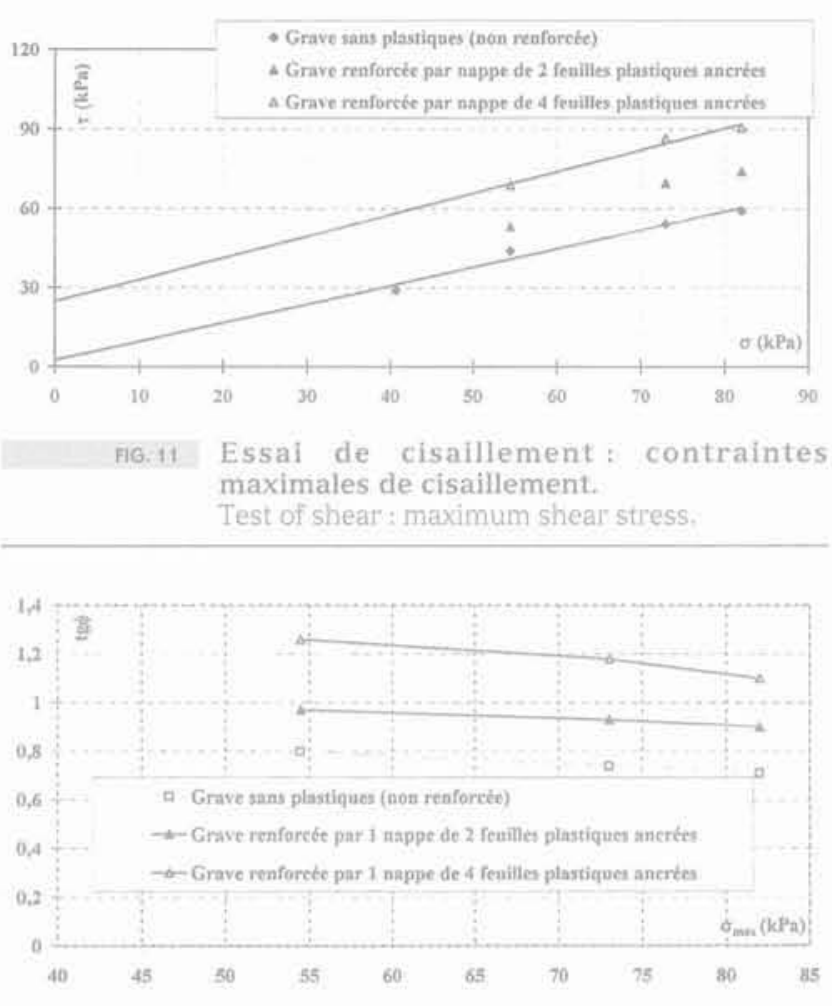

Fig. 12 Variation du coefficient de frottement apparent en fonction de la contrainte normale (influence du mode d'ancrage). Variation of the apparent friction coefficient according to the normal stress (influence of the anchoring mode)

\section{2}

\section{Calcul du gain de cohésion}

A la rupture, on suppose que le sol est en équilibre plastique le long du plan de cisaillement (Fig. 6). Les différentes forces s'exerçant sur le prisme ABCD (ta demi-boîte $n^{\circ}$ 2) (Fig. 13) sont :

$\mathrm{C}_{2}$; la surchage appliquée sur la demi-boite $n^{\circ} 2$;

$\mathrm{P}_{\mathrm{g}}$ : le poids propre de la grave ;

$R$ : la réaction du sol sur le plan de rupture AD. D'après I'hypothèse d'état de rupture du sol, cette réaction est inclinée d'un angle $\phi$ par rapport à la normale au plan de rupture:

$T:$ l'effort de cisaillement effectif;

$\mathrm{T}_{\mathrm{p}}$ : la force maximale mobilisée dans la nappe plastique :

N : l'effort normal dû aux charges verticales (surcharges, poids de la grave).

L'équilibre de ces forces à la rupture représenté par le diagramme de la figure 14 , permet d'écrire:

$$
\mathrm{T}=\left(\mathrm{N}+\mathrm{T}_{\mathrm{p}}\right) \mathrm{tg} \phi
$$

La surface de cisaillement $S_{i}$ varie en fonction du déplacement:

$$
\mathrm{T} / \mathrm{S}_{\mathrm{c}}=\left(\mathrm{N} / \mathrm{S}_{\mathrm{c}}+\mathrm{T}_{\mathrm{p}} / \mathrm{S}_{\mathrm{c}}\right) \operatorname{tg} \phi \Rightarrow \tau=\sigma \operatorname{tg} \phi+\left(\mathrm{T}_{\mathrm{p}} / \mathrm{S}_{\mathrm{c}}\right) \operatorname{tg} \phi
$$

En identifiant la relation (6) avec la loi de Coulomb $(\tau=\sigma \operatorname{tg} \phi+C)$, on peut déduire l'expression de la cohésion:

$$
\mathrm{C}=\left(\mathrm{T}_{\mathrm{p}} / \mathrm{S}\right) \operatorname{tg} \phi
$$

Si la nappe est constituée de $\mathrm{n}$ feuilles de matières plastiques de largeur $\mathrm{b}$ ayant une résistance de traction à la rupture $T_{p r}$ alors on pourra écrire : où :

$$
\Delta \mathrm{C}=\mathrm{n} \cdot \mathrm{T}_{\mathrm{pr}} \mathrm{b} \cdot \operatorname{tg} \phi / \mathrm{S}_{\mathrm{c}}
$$

n est le nombre de feuilles plastiques

$T_{\text {pr }}$ est la résistance de traction à la rupture d'une feuille plastique :

b est la largeur des feuilles plastiques;

$\$$ est la surface de cisaillement;

$\phi$ est l'angle de frottement interne de la grave.

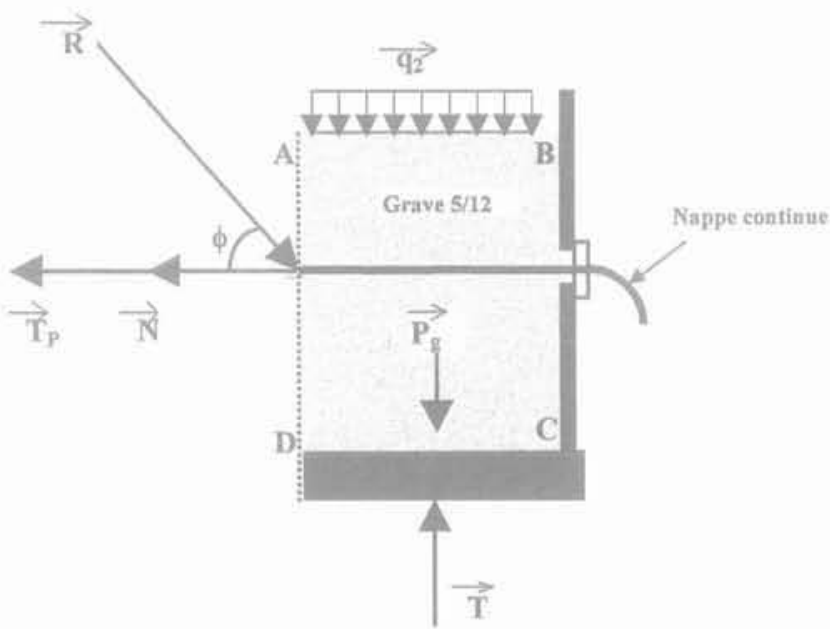

FG. 13 Bilan des forces extérieures agissant sur le sol contenu dans la demi-boîte mobile. External force result that act on the soil within half-box moving.

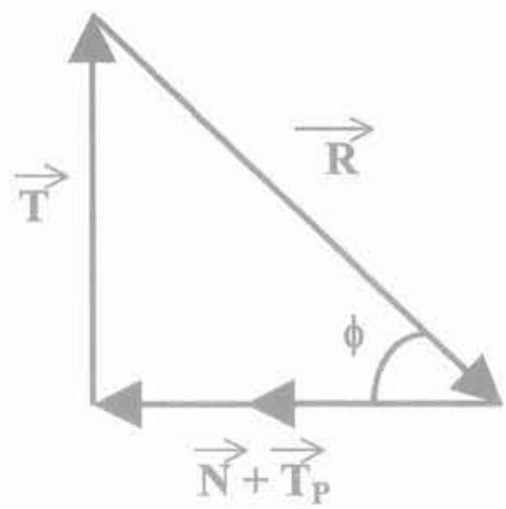

FIG. 14 Diagramme d'équilibre des forces. Balance of forces diagram.

Le tableau I compare les valeurs de $\Delta C$ calculées aux valeurs mesurées lors des essais de cisaillement. Compte tenu des précisions de mesures, la concordance est jugée acceptable.

TABLEAUI Valeurs du gain de cohésion théorique et expérimentale apporté par la présence des nappes continues.

Experimental and theoretical values of the cohesion gain due to continuous layers.

\begin{tabular}{l|c|c}
\hline Types d'essai & $\Delta \mathrm{C}_{\mathrm{cop}}(\mathrm{kPa})$ & $\Delta \mathrm{C}_{\mathrm{th}}(\mathrm{kPa})$ \\
$\begin{array}{l}\text { Grave renforcêe par nappe } \\
\text { de deux feuilles }\end{array}$ & 9 & 13 \\
\hline $\begin{array}{l}\text { Grave renforcée par nappe } \\
\text { de quatre feuilles }\end{array}$ & 22 & 25 \\
\hline
\end{tabular}




\section{Renforcement par des nappes discontinues}

\section{Experimentation \\ Expérimentation}

L'étude des nappes discontinues est conduite comparativement avec celle des nappes continues, en faisant varier deux paramètres: la dimension des éléments et leur répartition en masse. L'objectif de ces essais est de trouver une relation entre l'apport en cohésion des nappes discontinues et l'apport des nappes continues afin de pouvoir caractériser les nappes discontinues par une certaine résistance à la traction $T_{\text {p }}$ qui est calculée à partir de la formule 8 :

$$
T_{p}=\Delta C_{\exp } S / b \cdot \operatorname{tg} \phi
$$

Contrairement à l'approche par nappe continue cette résistance à la traction $T$, ne correspond pas à la rupture d'un élément mais à la rupture de la nappe par glissement des éléments les uns par rapport aux autres. Le tableau II donne l'ensemble des résultats oủ l'on constate évidemment la croissance de $T_{\text {, en fonction de }}$ l'augmentation de la taille des éléments.

L'évolution du rapport de la contrainte de cisaillement maximale $\left(\tau_{0 \max } / \tau_{\max }\right)$ en fonction de la forme géométrique des morceaux plastiques est représentée sur la figure 15 ( $\tau_{\text {max }}$ et $\tau_{\text {max }}$ sont des contraintes de cisaillement maximale du gravier renforcé et non renforcé). Pour une dimension donnée, T croit avec l'augmentation de la masse jusqu'à une valeur palier (Fig. 16). Les essais de graves renforcées par nappe, de longueur de recouvrement de moins de $10 \mathrm{~cm}$, ont été exclus car on estime que l'apport du renforcement dans ce cas est négligeable. Pour mieux approcher la réalité des renforts par déchets, des essais ont été également effectués avec des nappes formées de sacs d'emballage souples.

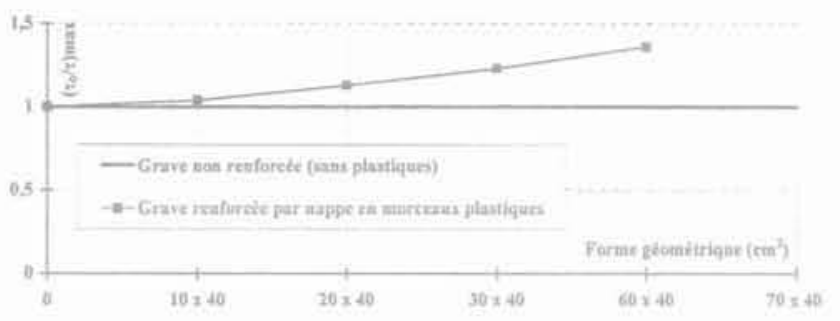

FIG. 15 Evolution du rapport de la contrainte de cisaillement $\left(\tau_{0} \max / \tau_{\text {man }}\right.$ fonction de la forme géométriqué des morceaux plastiques $\left(\sigma=54,5 \mathrm{kPa}\right.$ et $\left.\mathrm{M}_{0}=1 \mathrm{~kg} / \mathrm{m}^{2}\right)$. Evolution of the maximum shear tensile strength ratio according to the geometrical form of plastic pieces $\left(\sigma=54.5 \mathrm{kPa}\right.$ and $\mathrm{M}_{\mathrm{a}}=$ $\left.1 \mathrm{~kg} / \mathrm{m}^{2}\right\}$.

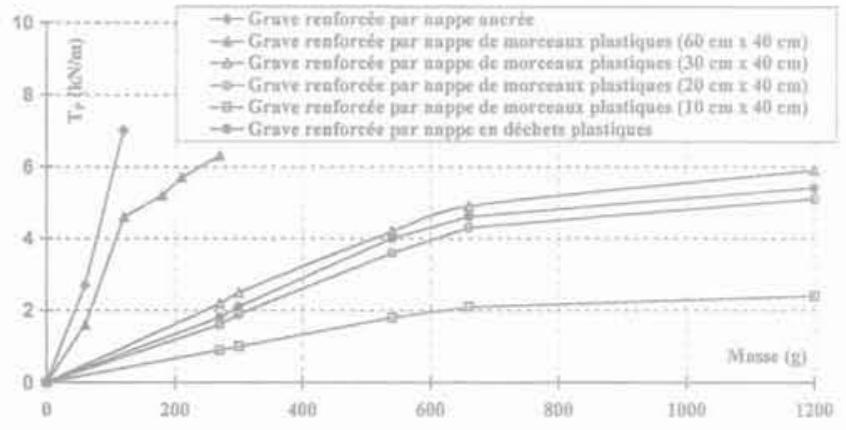

FiG.16 Résistance à la traction en service de la nappe en matières plastiques.

The tensile strength for the layer of plastic materials.

TABLAUII Valeurs de résistances à la traction en service des nappes plastiques en morceaux. Values of the tensile strength for plastic layers in pieces.

\begin{tabular}{|c|c|c|c|c|c|c|c|c|c|}
\hline & Masse (g) & (6) & 120 & 210 & 270 & 300 & 540 & 660 & 1200 \\
\hline & $\begin{array}{c}\text { Masse surfacique M } \\
\left.\text { (t.g } \mathrm{m}^{2}\right)\end{array}$ & 0,25 & 0,5 & 0.87 & 1,1 & 1,2 & 22 & 2.7 & 5 \\
\hline $\begin{array}{l}\text { Grave renforcée par nappe } \\
\text { de morceau } x \text { plastiques } \\
(60 \mathrm{~cm} \times 40 \mathrm{~cm})\end{array}$ & $\begin{array}{c}\Delta C_{m, 1}(\mathrm{kPa}) \\
(\mathrm{kN} / \mathrm{m})\end{array}$ & $\begin{array}{l}5,1 \\
1,6\end{array}$ & $\begin{array}{r}14,8 \\
5,2\end{array}$ & $\frac{20}{6,3}$ & & & & & \\
\hline $\begin{array}{l}\text { Grave renforcée par nappe } \\
\text { de morceaux plastiques } \\
(30 \mathrm{~cm} \times 40 \mathrm{~cm})\end{array}$ & $\begin{array}{c}\Delta C_{\mathrm{exp}}(\mathrm{kPa}) \\
T_{p=}=\frac{\Delta \mathrm{C}_{\mathrm{epp}} \mathrm{S} / \mathrm{b} \cdot \mathrm{tg} \phi}{(\mathrm{kN} / \mathrm{m})}\end{array}$ & & & & $\begin{array}{l}7 \\
2.2\end{array}$ & $\begin{array}{l}8 \\
2,5\end{array}$ & $\begin{array}{r}13,3 \\
4,2\end{array}$ & $\begin{array}{r}15,6 \\
4,9\end{array}$ & $\begin{array}{r}18,7 \\
5,9\end{array}$ \\
\hline $\begin{array}{l}\text { Grave renforcée par nappe } \\
\text { de morceaux plastiques } \\
(20 \mathrm{~cm} \times 40 \mathrm{~cm})\end{array}$ & $\begin{array}{c}\Delta C_{e q+}(\mathrm{kPa}) \\
\mathrm{Tp}=\frac{\Delta \mathrm{C}_{\mathrm{mp}} \mathrm{Sc} / \mathrm{b} \cdot \mathrm{tg} \phi}{(\mathrm{kN} / \mathrm{m})}\end{array}$ & & & & $\begin{array}{l}5 \\
1.6\end{array}$ & $\begin{array}{l}6 \\
1.9\end{array}$ & $\begin{array}{r}11,4 \\
3,6\end{array}$ & $\begin{array}{r}13,6 \\
4.3\end{array}$ & $\begin{array}{r}16,2 \\
5.1\end{array}$ \\
\hline $\begin{array}{l}\text { Grave renporcée par nappe } \\
\text { de morceaux plastiques } \\
(10 \mathrm{~cm} \times 40 \mathrm{~cm})\end{array}$ & 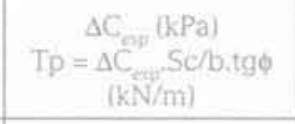 & & & & $\begin{array}{l}2,8 \\
0.9\end{array}$ & $\begin{array}{l}3,1 \\
1\end{array}$ & $\begin{array}{l}5,7 \\
1,8\end{array}$ & $\begin{array}{l}6,6 \\
2,1\end{array}$ & $\begin{array}{l}7,6 \\
2,4\end{array}$ \\
\hline $\begin{array}{l}\text { Grave renforcée par nappe } \\
\text { en déchets plastiques }\end{array}$ & $\begin{array}{c}\Delta \mathrm{C}_{\mathrm{cp}}(\mathrm{kPa}) \\
\mathrm{Tp}=\frac{\Delta \mathrm{C}_{\mathrm{sip}} \mathrm{Sc} / \mathrm{b} \cdot \operatorname{tg} \phi}{(\mathrm{kN} / \mathrm{m})}\end{array}$ & & & & $\begin{array}{l}5.7 \\
1.8\end{array}$ & $\begin{array}{l}6,6 \\
2,1\end{array}$ & $\begin{array}{c}12,7 \\
4\end{array}$ & $\begin{array}{r}14.6 \\
4.6\end{array}$ & $\begin{array}{r}17,1 \\
5,4\end{array}$ \\
\hline
\end{tabular}


TABLEAU III Valeurs des rapports équivalents des quantités de matières plastiques pour une résistance en service donnée Values of the tensile strength for plastic layers in pieces.

\begin{tabular}{|c|c|c|c|c|c|}
\hline \multirow{2}{*}{$\begin{array}{l}\mathrm{T}_{p} \\
(\mathrm{kN} / \mathrm{m})\end{array}$} & \multicolumn{5}{|c|}{$R_{E:}$} \\
\hline & $\begin{array}{l}\text { Grave renforcée } \\
\text { par nappe de MP } \\
\left(60 \times 40 \mathrm{~cm}^{2}\right)\end{array}$ & $\begin{array}{c}\text { Grave renforcée } \\
\text { par: nappe de MP } \\
\left(30 \times 40 \mathrm{~cm}^{2}\right)\end{array}$ & $\begin{array}{l}\text { Grave renforcée } \\
\text { par nappe de MP } \\
\left(20 \times 40 \mathrm{~cm}^{2}\right)\end{array}$ & $\begin{array}{l}\text { Grave renforcée } \\
\text { par nappe de-MP } \\
(10 \times 40 \mathrm{~cm})\end{array}$ & $\begin{array}{c}\text { Grave renforcée } \\
\text { par nappe } \\
\text { en déchets plastiques }\end{array}$ \\
\hline 2 & 2 & 6,8 & 8,1 & 16 & 7,8 \\
\hline 3 & 1,6 & 6,6 & 7.9 & & 6,9 \\
\hline 4 & 1,7 & 7,1 & 7,8 & & 7,5 \\
\hline 5 & 1,8 & 7,6 & 9,7 & & 7,8 \\
\hline
\end{tabular}

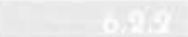

\section{Exploitation}

La courbe 16 permet de faire la correspondance entre la résistance à la traction des nappes discontinues et celle équivalente des nappes continues, Le rapport d'équivalence $R_{\mathrm{E}}$ (masse des morceaux des nappes discontinues/masse des feuilles des nappes continues) varie fortement avec d'une part la taille des éléments de renfort et d'autre part la résistance à la traction recherchée (tableau III). On peut noter que pour obtenir une continuité équivalente satisfaisante, la taille des éléments doit être au minimum de $30 \mathrm{~cm}$, et quand elle atteint $60 \mathrm{~cm}$, la valeur de $\mathrm{R}_{\mathrm{c}}$ est égale à 6 pour des résistances à la traction $T_{\text {de }} 8 \mathrm{kN} / \mathrm{m}$. Dans ce cas, on obtient des résultats tout a fait comparables avec certains géotextiles.

\section{7}

\section{Conclusion}

Les essais à plan de cisaillement vertical, effectués sur des échantillons de sols renforcés par des nappes continues ou discontinues, ont montré que :
- plus la quantité et la taille des matières plastiques augmentent, plus le chevauchement et le recouvrement entre les morceaux sont importants. Le comportement du renfort discontinu tend vers celui de la nappe continue; - la comparaison des valeurs du gain de cohésion théorique et expérimentale permet de quantifier la résistance de cisaillement effective apportée par les nappes discontinues.

L'étude comparative des essais de frottement et des essais d'arrachement a abouti aux remarques suivantes: - la valeur de l'angle de frottement d'interface graveplastique déterminée par l'essai d'arrachement est inférieure à celle obtenue lors des essais de frottement; - on note une certaine analogie qualitative de la variation du coefficient de frottement apparent en fonction de la contrainte normale pour les deux types d'essai; - l'essai de frottement a été adapté pour étudier le frottement lié à des propriétés d'interface. L'essai d'arrachement semble cependant mieux modéliser le comportement réel d'une nappe plastique ancrée.

Enfin, l'utilisation de déchets plastiques permet, d'une part, d'améliorer les caractéristiques mécaniques des sols et, d'autre part, de valoriser des déchets coûteux à éliminer.

\section{Bibliographie}

Bourdeau Y Kastner R. - "Etude de l'interaction sol-géotextile par essais d'arrachement is. C. R. Journées francotunisiennes Mécanique des sols : stabilité et renforcement des pentes, Paris. mai 1989, p. 39-45.

Coulet C., Rakotondramanitra J.D. Bacot J. - « Soil reinforcement making use of waste plastic materials study with large shear box machine x. VIIIth Nat. Conference on Soil Mecanics and Foundation Engineering, Wroclaw, Pologne, 1987.

Delmas P.. Gourc J.-P., Giroud J.-P. «Analyse expérimentale de l'interaction sol-géotextile 2. C. R. Colloque Int, sur le renforcement des sols, Paris, vol. $\mathrm{t}$. 1979, D. 29-34

Gourc J.-P.. Monnet J., Mommessin M. "Reinforced embankments on weak soil different theorical approches 1). Proc. 3rd International Conference on Geotextiles. Vienna, vol. 4. 1986, p. 1043-1049.

Ingold T.S. - « Friction testing, geomembranes ; identification and performance testing $n$. RILEM 103-MGH, friction tes ting, $1989,28 \mathrm{p}$.

Omine K., Ochiai H., Kato T. - « Effect of plastic wastes in improving cement treated soils 11, Environmental Gegtechnics, Kamon 1996, Rotterdam, Balkema. vol. II, 1996, p. 875-880.

Messas T. - a Étude des renforcements des sols par nappes discontinues en matières plastiques souples i. Thèse présentée à l'université Claude-Bernard de Lyon (UCBL), soutenue le 6 novembre 1997, pour obtenir le grade de Docteur, 1997.

Schlosser F. Jacobsen H.M., Juran 1. - " Le renforcement des sols 12 . Revue française de géotechnique, $n^{\circ} 29,1984$, p. 7-33. 